\title{
EVALUACIÓN DE LA INVERSIÓN EN OBRAS POR IMPUESTO Y SU IMPACTO SOCIO ECONÓMICO EN LA REGIÓN DE TACNA, PERIODO $2009-2016$
}

\author{
EVALUATION OF THE INVESTMENT IN WORKS FOR TAX AND ITS \\ ECONOMIC SOCIAL IMPACT IN THE TACNA REGION, PERIOD 2009 - 2016
}

NATALY JENNIFER BENDEZÚ MELÉNDEZ 1

\section{Resumen}

Determinar la relación de la inversión en obras por impuesto y el impacto socio económico en la Región de Tacna, Periodo 2009 - 2016. Se utilizó un diseño correlacional - cuantitativo, empleando la revisión documental del Ministerio de Economía y Finanzas, Prolnversión, Obras por Impuesto, así como un cuestionario estructurado, aplicado a la población beneficiaria de obras por Impuesto en la Región de Tacna. La relación entre la inversión en obras por impuesto y el impacto socio económico en la Región de Tacna es significativa. Asimismo, la inversión en obras por impuesto desde el 2009 al 2016 fue moderada, en cuanto al impacto socio económico entre el 2009 y el 2016 fue favorable. Finalmente se verificó que la inversión en obras por impuesto en la Región de Tacna, mejoró su crecimiento desde el año 2014. La relación entre la inversión en obras por impuesto y el impacto socio económico en la Región de Tacna, Periodo 2009 2016 si es significativa. Puesto que al analizar estadísticamente ambas variables se observa que la obras por impuesto inciden de forma directa en el impacto socioeconómico en la Región de Tacna.

Palabras clave: Inversión en obras por impuesto, impacto socio económico.

\section{Abstract}

Objective: To determine the relation of the investment in works by tax and the socioeconomic impact in the Region of Tacna, Period 2009 - 2016. Methodology: A correlational - quantitative design was used, using the documentary review of the Ministry of Economy and Finance, Prolnversión, Obras por Impuesto, as well as a structured questionnaire, applied to the beneficiary population of works for Tax in the Region of Tacna.

Results: The relation between the investment in works by tax and the socio-economic impact in the Region of Tacna is significant. Likewise, the investment in works for tax from 2009 to 2016 was moderate, as far as the socio-economic impact between 2009 and 2016 was favorable. Finally it was verified that the investment in works by tax in the Region of Tacna, improved its growth from the year 2014. Conclusion: the relationship between investment in works by tax and socioeconomic impact in the Region of Tacna, Period 2009 - 2016 if significant. Since the statistical analysis of both variables shows that the works by tax have a direct impact on the socio-economic impact in the Region of Tacna.

Keywords: Investment in works by tax, socioeconomic impact.

\section{INTRODUCCIÓN}

La Ley N²9230 "Obras por Impuestos", brinda a la empresa privada la oportunidad de financiar obras públicas y recuperar la inversión realizada a través de la reducción de su pago de impuesto a la renta. Esto genera un aumento en la eficiencia de sus programas de responsabilidad social con obras de gran impacto, contribuyendo a mejorar la percepción que tiene la población de la empresa.

1 MAESTRO EN INGENIERÍA CIVIL CON MENCIÓN EN GERENCIA DE LA CONSTRUCCIÓN 
El esquema de inversión de obras por impuestos ha venido creciendo sostenidamente desde su creación en 2009. A la fecha, a nivel nacional se tienen 230 proyectos adjudicados y concluidos, de los cuales en Tacna se han realizado únicamente 11 con una inversión de 209 millones. Los departamentos de Arequipa, Ancash, Piura y Tacna lideran el ranking de en monto de inversión en forma descentralizada.

Esta norma brinda una muy buena opción para contribuir al desarrollo de la comunidad; sin embargo, no está siendo aprovechada por los gobiernos locales. Este mecanismo surgió para incentivar la participación activa del sector privado en proyectos de infraestructura pública necesarios para mejorar las condiciones de vida de la población y la competitividad del país, habiendo promovido una serie de obras dentro de infraestructura pública básica, como redes de agua y desagüe, vías, centros de salud, colegios, entre otras. Sin embargo, a pesar del tiempo transcurrido y los evidentes beneficios para la empresa privada y las zonas donde se han desarrollado obras, no ha sido posible hasta ahora aprovechar en todo su potencial este innovador mecanismo. En las 30 municipalidades de la Región de Tacna, se puede apreciar que sólo 08 municipios han optado por ejecutar sus proyectos bajo la modalidad de obras por impuesto, por lo que 22 municipios de esta región no han aprovechado aún la inversión de la empresa privada.

El éxito de un sistema no sólo radica en su aplicación, sino en la necesidad de hacerlo de la mejor manera, además permite una gestión más eficaz y eficiente, buscando optimizar los recursos, aprovechar las sinergias mutuas del beneficio del ciudadano y beneficiando a la empresa a mejorar su competitividad y buena imagen. Harry Chang Yong, en el 2015 en la Revista Tiempo de Opinión de la Universidad ESAN, hace mención que en los últimos años, el componente más importante del crecimiento del Perú ha sido y lo seguirá siendo la inversión, tanto pública como privada.

Otero, J \& Núñez, M (2014). Realizan un estudio de caso: los proyectos de obras por impuestos como parte de la política de responsabilidad social empresarial (RSE) del Banco de Crédito (BCP). Sin embargo, no se tuvo acceso a mayor información, por lo que no se cuenta con los resultados de dicha tesis. Así también Cruz, L, Cuti, R, García, S, Mujica, M, Valdez, C \&Zamata, G (2015). Realizan un Análisis de los Impactos
Directos de una obra por Impuesto: Caso Puente Chilina", determinando que los impactos directos positivos de una obra por impuesto, son limitados, debido al esporádico uso de la obra, insatisfacción por parte de la población respecto a la construcción del puente por la ausencia de rutas del transporte público que hagan uso del puente, la principal ventaja de esta construcción es la reducción del tiempo de transporte, siendo ésta sólo aprovechada por la mitad de las poblaciones de los distritos de Alto Selva Alegre y Cayma, habiéndose logrado una interconexión entre los distritos, logrando reducir el tiempo de traslado de un distrito a otro, beneficiando a la población. En el 2015, en la Universidad Nacional de Trujillo, Melgarejo, R \& Sare, C (2015). Observan la contribución del régimen de obras por impuestos de la ley N²9230 en la eficiencia y ejecución de proyectos de inversión pública, estudiando los principios de eficiencia, transparencia y ética en la ejecución de proyectos de inversión pública en el gobierno local, planteado como objetivos analizar si el régimen de obras por impuesto de la ley N²9230 ha cumplido con el propósito de ejecutar con celeridad los proyectos de inversión pública en infraestructura y determinar si este régimen permite la eficiencia en la ejecución de proyectos de inversión pública de impacto local. Albújar, $A$, Santa Cruz, E,Albújar, J,Gómez, E,Quezada, K\& Terrones, S. (2016) demostraron que, existen factores influyentes como falta de capacidad técnica y especializada, barreras burocráticas, presencia de sobrecostos y riesgos en la modalidad Obras por Impuestos. Sin embargo, estos factores negativos no son suficientes para disuadir a las empresas de la decisión de participar en esta modalidad $y$, al final de su experiencia, en un $96 \%$ de los casos, calificarla como excelente o buena y expresan que volverían a financiar obras a través de esta modalidad, por lo que se infiere que de igual manera no influirían en la decisión de las empresas que aún no han apostado por financiar proyectos a través de Obras por Impuestos. El análisis del perfil de las empresas que a la fecha han invertido en la modalidad de Obras por Impuestos y, luego del análisis de sus ventas, utilidad neta, rubro y radio de acción, entre otros factores, se ha determinado que todas han financiado obras en la zona donde ejercen influencia. Igualmente queda claro que las empresas privadas prefieren ejecutar proyectos con los gobiernos locales, antes que con los gobiernos regionales, por su deseo de crear un buen clima con el gobierno local y la población allí 
donde ejercen influencia de manera directa y mejorar la relación empresa-comunidad y empresa-gobierno local. Al final concluyó que el más conveniente es que Prolnversión tenga un nuevo papel protagónico dentro del proceso ya que cuenta con el personal idóneo y la capacidad técnica y especializada para gestionar y ejecutar proyectos de infraestructura pública. Sin embargo, considerando que esta alternativa podría generar una saturación de la capacidad de Prolnversión se planteó la posibilidad de establecer un monto mínimo de inversión de las obras que se sometan al ámbito de esta entidad.

\section{IMPACTO SOCIO ECONÓMICO}

En Noviembre del 2015, durante el primer Congreso Internacional de Infraestructura y Desarrollo (CIID), fue presentado el estudio denominado "Un plan para salir de la pobreza Plan Nacional de Infraestructura 2016 - 2025" el cual fue realizado por la Escuela de Gestión Pública de la Universidad del Pacífico, encargado por AFIN; en dicho estudio se dio a conocer la brecha para el Perú de infraestructura 20162025. La brecha total $2016-2025$ proyectada es de 160 mil millones de dólares, compuesta por 70,000 mil millones de brecha a mediano plazo 2016-2020, y más 90 mil millones de brecha a largo plazo 2021-2025. Los sectores con las brechas más grandes son transportes con $36 \%$, energía con $19 \%$, telecomunicaciones con $17 \%$ y salud con $12 \%$ de la brecha total. La brecha $2012-$ 2021, al cerrarse, nos llevaría el 2021 al nivel de infraestructura promedio de América Latina; mientras que la nueva brecha de mediano plazo 2016-2020, proyectada en 70,000 millones, al cerrarse nos pondría el 2020 al nivel del promedio en infraestructura de la Alianza del Pacífico. Según el reporte de competitividad global 20152016 del Foro Económico Mundial, en términos de puntaje de infraestructura la Alianza del Pacífico promedia 4.0 y Latinoamérica promedia 3.6. Perú tiene 3.5 puntos y el máximo puntaje eficiente es 7.0. La brecha actualizada al 20162025 incluye sectores sociales como Salud y Educación que suman 23,500 millones de dólares por $15 \%$ de la brecha total, e incluye también Agua y Saneamiento con 12,300 millones y $8 \%$ de la brecha total. El objetivo trazado para el cierre de la brecha $2016-2025$ es en el largo plazo, al 2025, alcanzar al promedio en infraestructura de una muestra de países asiáticos (China, Japón, Corea del Sur, Filipinas, Vietnam, Indonesia, Tailandia y otros).

El monto de la brecha no se halla sumando montos de una lista de proyectos, sino calculando la inversión necesaria para alcanzar niveles de indicadores por sectores, como camas de hospital por cada 1000 habitantes, consumo de energía per cápita, o kilómetros de carreteras por habitante, similares a los indicadores de los grupos de países referentes, la Alianza del Pacífico y la muestra de países asiáticos, respectivamente.

Como el plan se proyecta a 10 años, según el estudio realizado para AFIN, se necesitaría invertir en infraestructura un promedio de $8 \%$ del $\mathrm{PBI}$ anual, cada año, y eso aún no se ha logrado en el país. Pero se puede intentar e ir mejorando los procesos hasta alcanzarlo.

Consolidar la brecha horizontal y vertical no puede realizarse como una suma simple, dado que no son totalmente independientes. Siempre que la brecha horizontal represente una exigencia para el país, esta debería incorporar parte de la brecha vertical. De este modo, y a pesar de que ambas brechas representan requerimientos de infraestructura que aparecen por distintos motivos, ambas deben revisarse por separado.

Es razonable pensar que los requerimientos reales de un país se encuentren fuertemente ligados a la brecha vertical de infraestructura que refleja las presiones de demanda, y que esta demanda de infraestructura pueda sufrir también saltos discretos denominados impulsores de demanda, que tienen como objetivo alcanzar a un grupo de países. Un impulsor típico de demanda por infraestructura está asociado a una voluntad política del gobierno por garantizar un mayor nivel de infraestructura en todos los casos. Como cada gobierno de turno tiene como prioridad la mejora en el bienestar de la población y la reducción de la pobreza, es razonable suponer que se impulse de manera sostenida la inversión en infraestructura considerando no sólo las demandas actuales, sino la posición relativa del país con respecto al resto del mundo. 
INVERSIÓN EN OBRAS POR IMPUESTO

La infraestructura cumple un papel trascendental en el desarrollo de las regiones, al generar beneficios que impactan de forma directa o indirecta sobre la economía en general, circunstancia corroborada por distintos estudios técnicos y académicos que revelan la existencia de diferencias marcadas en aquellas familias que cuentan con acceso a infraestructura y aquellas que no, ya que en el caso de estas últimas su ámbito de trabajo, en su mayor parte, se circunscribe a realizar labores de campo. Una correcta provisión de servicios públicos requiere de un adecuado desarrollo de infraestructura que la sustente y que permite su prestación concreta; sin embargo, a pesar de que en la actualidad ha habido avances considerables, la demanda por infraestructura se encuentra aún muy lejos de estar satisfecha. Hecho que se debe en gran medida a que en los últimos años las decisiones de los inversionistas se encuentran influenciadas por los riesgos tanto financieros como regulatorios, lo que ha llevado al empresariado a asumir mayor cautela en la toma de decisiones respecto de los proyectos a los cuales orientan su capital. En la actualidad son diversos los servicios en los que se requiere de infraestructura para lograr los objetivos que definen la política nacional, como la inclusión social y el fomento de la inversión privada, entre otros. La modalidad de Obras por Impuestos, es una herramienta de política creada en el Perú, que no tiene antecedentes en otros países. Desde su dación a la fecha, el marco normativo se ha complementado permitiendo su agilidad y buscando generar confianza entre los actores públicos y privados que participan en su adecuada aplicación.

\section{OBJETIVOS}

Determinar el nivel de inversión del sector privado en el sector público, y su relación con la ejecución de obras por impuesto en la Región de Tacna.
Establecer la relación que existe entre la ejecución de obras por impuesto y la población beneficiada de la Región de Tacna.

\section{METODOLOGÍA}

Se ha realizado una Investigación descriptiva. El ámbito de la investigación es la Región de Tacna. Se tomaron como unidad de estudio, las zonas donde se cuenta con inversión en obras por impuesto en la Región de Tacna: Municipalidad
Provincial Jorge Basadre, Municipalidades Distritales de llabaya, Pocollay, Ciudad Nueva, Coronel Gregorio Albarracín Lanchipa, Ite, Camilaca y Quilahuani.

\section{RESULTADOS}

Durante el periodo de estudios, en los gobiernos locales se tienen obras por impuesto con una inversión total de S/209'119,460.04.
GRAF. 01: monto de Inversión en Obras por Impuesto por cada localidad de la Región de Tacna

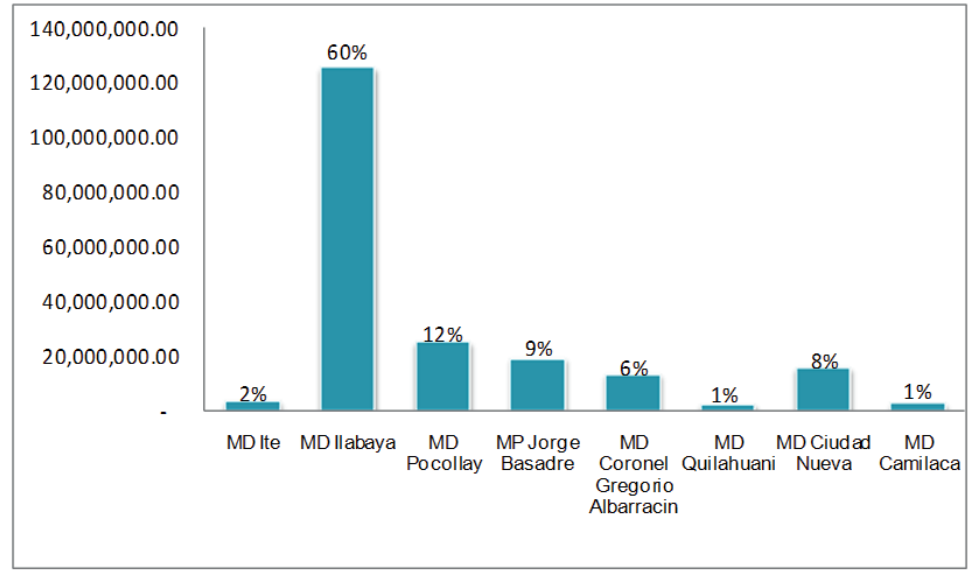


Durante el periodo de estudios, en la Provincia de Jorge Basadre se ha ejecutado el $71 \%$ del monto de la inversión de obras por impuesto en la Región de Tacna con una inversión de 149.1 millones de soles, seguido por la Provincia de Tacna con un $26 \%$ con una inversión del 54.7 millones de soles, la Provincia de Candarave con un $3 \%$ con una inversión de 5.3 millones de soles, y en la Provincia de Tarata no se cuenta con inversión en obras por impuesto. Los gobiernos locales han invertido en total en 11 proyectos, en los sectores de transporte, saneamiento, cultura y telecomunicaciones. Se tiene que en el Sector de Transporte se ha invertido en 08 proyectos, siendo éste el tipo de obra más utilizada en la región con un $73 \%$; así también se ha ejecutado 01 obra de telecomunicaciones, 01 de saneamiento y 01 de cultura.

Son 03 las Empresas que han financiado obras por impuesto; la Empresa Southern Perú Cooper Corporation con $92 \%$, es la que más ha invertido en obras por impuesto por un monto de S/ 192'015,133.78, seguido por el Banco de Crédito del Perú con $6 \%$ de inversión por un monto de 13.5 millones de soles y seguido por el Telefónica del Perú S.A.A. con $2 \%$ de inversión por un monto de 3.6 millones de soles.

La Empresa Southern Perú Cooper ha invertido un $66 \%$ en la Municipalidad de llabaya con un monto de inversión de $S / 126,030,388.25$, seguido por un $13 \%$ en la Municipalidad Distrital de Pocollay con un monto de S/ 25,314,029.83, un $10 \%$ en la Municipalidad Provincial de Jorge Basadre con un monto de $S / 19,480,976.90$, un $8 \%$ en la Municipalidad Distrital de Ciudad Nueva con un monto de $S / 15^{\prime} 918,782.05$, un $1 \%$ en la
Municipalidad Distrital de Camilaca con un monto de $S / 2 ' 845,187.66$ y un $1 \%$ en la Municipalidad Distrital de Quilahuani con un monto de inversión de $S / 2 ' 425,769.09$. La empresa BCP ha invertido en obras por impuesto únicamente en la Municipalidad Distrital Coronel Gregorio Albarracín Lanchipa, con una inversión de S/13'477,959.53. La empresa Telefónica del Perú S.A.A. ha invertido en la Municipalidad Distrital de Ite, con una inversión de S/3'626,366.73.

De la encuesta dirigida a la población de las localidades beneficiadas con obras por impuesto, se evidencia que el $52.1 \%$ de la población conoce acerca de las obras por impuesto que se han venido ejecutando en localidad, el $32.9 \%$ conoce la proveniencia de los recursos económicos para las obras por impuesto, mientras que el $67.1 \%$ no tiene conocimiento.

El $41.1 \%$ de la población sabe qué empresas financian las obras por impuesto de su localidad, y de estas el $23.3 \%$ la califica de regular, el $36.7 \%$ de buena y el $40.0 \%$ de muy buena la inversión.

Respecto a cómo califica la participación del Municipio en la promoción de la inversión en obras por impuesto, el $11.0 \%$ califica de deficiente, el $61.6 \%$ de regular, el $21.9 \%$ de buena y el $5.5 \%$ de muy buena.

El $72.6 \%$ siente que ha mejorado su calidad de vida con la ejecución de estas obras por impuesto, el $11.00 \%$ siente que no ha sido así, mientras que el $16.4 \%$ no sabe y/o no opina. Respecto al impacto económico, el $9.6 \%$ lo califica como negativo, el 75.3 como un impacto positivo, mientras que el $15.1 \%$ no sabe o no opina.

\section{DISCUCIÓN}

La modalidad de Obras por Impuesto, fue creado en el Perú a fin de ejecutar proyectos a cargo de los gobiernos locales, gobiernos regionales, con financiamiento de la empresa privada, siendo esta modalidad es una gran alternativa que en la Región de Tacna se debería emplear, considerando que para el año 2016, según Prolnversión tuvo un monto disponible para la ejecución de obras por impuesto de S/ $552 ' 046,637.81$, sin embargo solo se ejecutó y/o comprometió un monto de S/21'189,738.80, es decir el $3.84 \%$ del presupuesto disponible, quedando sin ejecutarse $S / 530 ' 856,899.01$ que representa el $96.16 \%$ del presupuesto disponible para obras por impuesto. Durante el periodo de estudio (2009 - 2016), se verifica el nivel de inversión del Sector Privado en las localidades de la Región de Tacna, bajo la modalidad de obras por impuesto, con un monto de inversión de $S / 209 ' 119,460.04$. Se verifica que la ejecución de obras por impuesto ha beneficiado significativamente a la población de la Región de Tacna, ya que en gran medida han mejorado su calidad de vida, con un impacto económico positivo, siendo satisfactoria la calificación por parte de la población beneficiaria.

El nivel de inversión del sector privado en el sector público, se relaciona directamente con la ejecución de obras por impuesto en la Región de Tacna y ha beneficiado significativamente a la población de la Región de Tacna. La propuesta de 
la presente investigación, buscar contribuir a acelerar la inversión a través de una modalidad que otorgue seguridad tanto a la Empresa como al Gobierno Local/Regional. Por esta razón, es necesario que Prolnversión cumpla un papel protagónico, promocionando los proyectos priorizados por los Gobiernos Locales y Regionales, para ello, Prolnversión en coordinación con los Gobiernos Locales y/o Regionales, deberá elaborar un Plan Anual de Inversión bajo la modalidad de Obras por Impuesto por cada Departamento. A fin de considerar los montos en dicho plan anual, se tomará en cuenta los límites para la emisión de los CIPRL de los Gobiernos Regionales y locales por cada año, es así que se formula una estimación que propone como meta anual a ejecutar el $30 \%$ del monto total acumulado de los límites de CIPRIL de los Gobiernos Regionales y locales de cada año, los cuales son publicados por el MEF en marzo de cada año.

El Gobierno Regional y los Gobiernos Locales deberían hacer uso del presupuesto disponible para la ejecución de obras por impuesto, que para el año 2016 ascendió a S/ 552'046,637.81 para la
Región de Tacna, a fin de cubrir la brecha de infraestructura existente. El Gobierno Regional así como los Gobiernos Locales de Tacna, que tengan previsto la ejecución de obras por impuesto, deben priorizar proyectos que satisfagan las necesidades más urgentes de la población y se beneficie a la mayor población, para lo cual se recomienda realizar las siguientes acciones: Se debe realizar un estudio detallado de la brecha en infraestructura que se requiere cubrir en la Región de Tacna, a fin de priorizar los proyectos; planificar la inversión en infraestructura, tomando en cuenta la brecha con la que se cuenta en la Región de Tacna, con planes de infraestructura integrados con las necesidades logísticas y con la visión estratégica de crecimiento de la Región de Tacna y se debe crear un Plan Regional de Infraestructura en coordinación con el sector privado considerando la brecha de infraestructura con la que se cuenta. Además, se debe fortalecer las capacidades del sector público con la finalidad de impulsar la inversión privada, en proyectos prioritarios, dotando de capacidades al sector público (gobierno regional y local), a fin de enfrentar con éxito los procesos de inversión pública y privada.

Presentado: Marzo 2017

Aceptado: Junio 2017

\section{REFERENCIAS BIBLIOGRÁFICAS}

AFIN (2015). "Estudio de Régimen de Obras por Impuestos", Asociación para el Fomento de la Infraestructura Nacional, Lima, Perú.

AFIN (2016). "Un Plan para salir de la pobreza - Plan Nacional de Infraestructura 2016 - 2025", Asociación para el Fomento de la Infraestructura Nacional, Escuela de Gestión Pública de la Universidad del Pacífico, Lima, Perú.

Albújar, A, Santa Cruz, E,Albújar, J,Gómez, E,Quezada, K\& Terrones, S. (2016) Libro "Obras por Impuestos: factores que promueven la participación de la empresa privada". Lima, Universidad ESAN.

Asociación para el fomento de la infraestructura Nacional AFIN (Octubre 2015), Publicación "Plan Nacional de Infraestuctura 2016 - 2025".

Cruz, L, Cuti, R, García, S, Mujica, M, Valdez, C \&Zamata, G (2015). Investigación de Pregrado "Análisis de los Impactos Directos de una obra por Impuesto: Caso Puente Chilina". Universidad Nacional San Agustín de Arequipa, Arequipa, Perú.

Decreto Legislativo $N^{\circ} 1250$, publicado en el Peruano el 30 de noviembre del 2016.

Decreto Supremo No 409-2015-EF. Reglamento de la Ley $N^{\circ} 29230$, Ley que impulsa la inversión pública regional y local con participación del sector privado, y del artículo 17 de la Ley $\mathrm{N}^{\circ}$ 30264 , Ley que establece medidas para promover el crecimiento económico, Diciembre del 2015

Ley N²9230. Ley que Impulsa la Inversión Pública Regional y Local con Participación del Sector Privado, Mayo del 2008.

Melgarejo, R \& Sare, C (2015). Tesis de pregrado "Contribución del régimen de obras por Impuestos de la Ley $N^{\circ} 29230$ en la eficiencia y ejecución de proyectos de inversión pública". Universidad Nacional de Trujillo, Trujillo, Perú.

Otero, J \& Núñez, M (2014). Tesis de pregrado "Estudio de caso: los proyectos de obras por impuestos como parte de la política de responsabilidad social empresarial (RSE) del Banco de Crédito (BCP)". Universidad Pontificia Universidad Católica del Perú, Lima, Perú.

Pro-Inversión (2016). Obras por impuesto. http://www.obrasporimpuestos.pe

Revista Gestión (2015-2016). http://www.gestion.pe 